\title{
Precision Measurement of the Spin-Dependent Asymmetry in the Threshold Region of ${ }^{3} \overrightarrow{\mathrm{He}}\left(\vec{e}, e^{\prime}\right)$
}

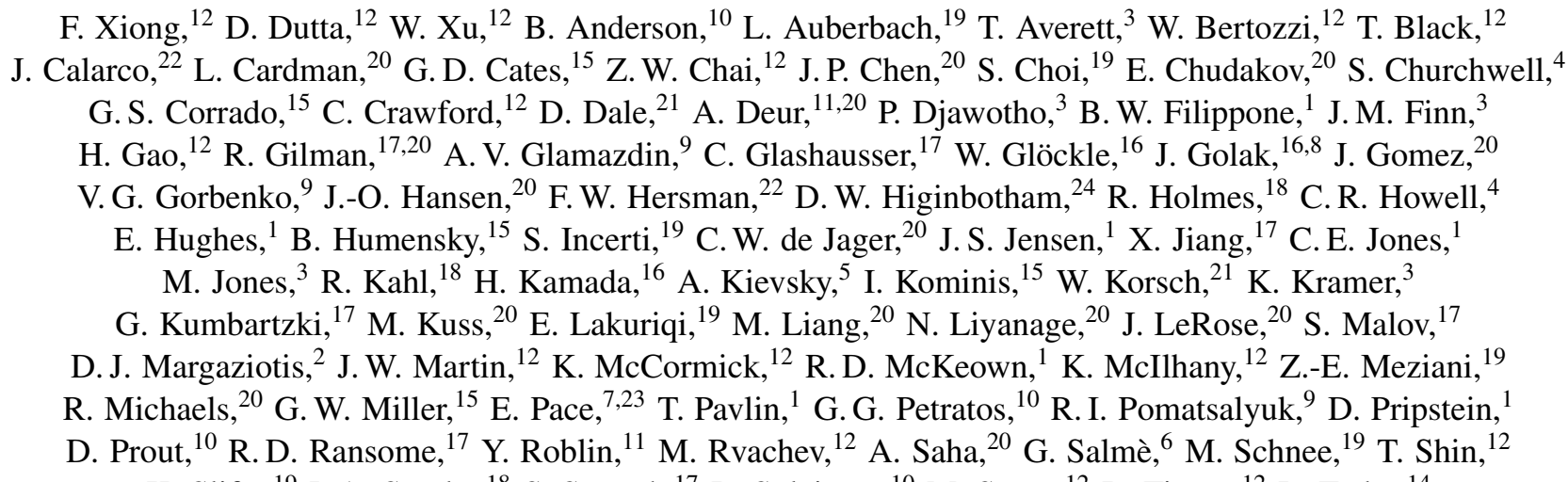

K. Slifer, ${ }^{19}$ P. A. Souder, ${ }^{18}$ S. Strauch, ${ }^{17}$ R. Suleiman, ${ }^{10}$ M. Sutter, ${ }^{12}$ B. Tipton, ${ }^{12}$ L. Todor,${ }^{14}$ M. Viviani, ${ }^{5}$ B. Vlahovic, ${ }^{13,20}$ J. Watson, ${ }^{10}$ C.F. Williamson, ${ }^{10}$ H. Witała, ${ }^{8}$

B. Wojtsekhowski, ${ }^{20}$ J. Yeh, ${ }^{18}$ and P. Żołnierczuk ${ }^{21}$

${ }^{1}$ California Institute of Technology, Pasadena, California 91125

${ }^{2}$ California State University of Los Angeles, Los Angeles, California 90032

${ }^{3}$ College of William and Mary, Williamsburg, Virginia 23187

${ }^{4}$ Duke University, Durham, North Carolina 27708

${ }^{5}$ INFN, Sezione di Pisa, 56010 S. Piero a Grado, Pisa, Italy

${ }^{6}$ INFN, Sezione di Roma, P. le A. Moro 2, I-00185 Roma, Italy

${ }^{7}$ INFN, Sezione Tor Vergata, Via della Ricerca Scientifica 1, I-00133 Rome, Italy

${ }^{8}$ Institute of Physics, Jagellonian University, PL-30059 Cracow, Poland

${ }^{9}$ Kharkov Institute of Physics and Technology, Kharkov 310108, Ukraine

${ }^{10}$ Kent State University, Kent, Ohio 44242

${ }^{11}$ LPC, Université Blaise Pascal, F-63177 Aubière, France

${ }^{12}$ Massachusetts Institute of Technology, Cambridge, Massachusetts 02139

${ }^{13}$ North Carolina Central University, Durham, North Carolina 27707

${ }^{14}$ Old Dominion University, Norfolk, Virginia 23508

${ }^{15}$ Princeton University, Princeton, New Jersey 08544

${ }^{16}$ Ruhr-University, D-44780 Bochum, Germany

${ }^{17}$ Rutgers University, Piscataway, New Jersey 08855

${ }^{18}$ Syracuse University, Syracuse, New York 13244

${ }^{19}$ Temple University, Philadelphia, Pennsylvania 19122

${ }^{20}$ Thomas Jefferson National Accelerator Facility, Newport News, Virginia 23606

${ }^{21}$ University of Kentucky, Lexington, Kentucky 40506

${ }^{22}$ University of New Hampshire, Durham, New Hampshire 03824

${ }^{23}$ Dipartimento di Fisica, Università di Roma “Tor Vergata," Rome, Italy

${ }^{24}$ University of Virginia, Charlottesville, Virginia 22903

(Received 1 July 2001; published 27 November 2001)

\begin{abstract}
We present the first precision measurement of the spin-dependent asymmetry in the threshold region of ${ }^{3} \mathrm{He}\left(\vec{e}, e^{\prime}\right)$ at $Q^{2}$ values of 0.1 and $0.2(\mathrm{GeV} / c)^{2}$. The agreement between the data and nonrelativistic Faddeev calculations which include both final-state interactions and meson-exchange current effects is very good at $Q^{2}=0.1(\mathrm{GeV} / c)^{2}$, while a small discrepancy at $Q^{2}=0.2(\mathrm{GeV} / c)^{2}$ is observed.
\end{abstract}

DOI: 10.1103/PhysRevLett.87.242501

Three-nucleon systems have been an excellent testing ground between theory and experiment in nuclear physics [1]. In the context of electromagnetic processes, exact nonrelativistic Faddeev calculations for both the ground state and the continuum of ${ }^{3} \mathrm{H}$ and ${ }^{3} \mathrm{He}$ have been carried out using a variety of modern nucleon-nucleon $(\mathrm{NN})$ potentials [2-4]. The exact treatment of final-state interactions
PACS numbers: $25.10 .+\mathrm{s}, 13.40 . \mathrm{Gp}, 24.70 .+\mathrm{s}$, 25.30.Fj

(FSI) in the Faddeev calculation results in a much improved description of unpolarized $p d$ capture and breakup channels $[2,4]$, as well as unpolarized electron scattering from the three-nucleon system [3]. This has provided important information on the nuclear ground-state structure and thus allows a deeper understanding of the underlying nuclear force. With the availability of polarized beams and 
polarized targets, it has become possible to study additional spin-dependent quantities. Polarized ${ }^{3} \mathrm{He}$ is an ideal target for such a study.

Polarized ${ }^{3} \mathrm{He}$ is also important as an effective neutron target $[5,6]$, because its ground-state wave function is dominated by the $S$ state in which the proton spins cancel and the nuclear spin is carried entirely by the neutron. The spin-dependent asymmetries are thus sensitive to the neutron electromagnetic form factors in the vicinity of the quasielastic peak of polarized electrons scattering from a polarized ${ }^{3} \mathrm{He}$ target [5-10]. Recently there has been significant progress in extracting neutron electromagnetic form factors from double-polarization electron $-{ }^{3} \mathrm{He}$ scattering experiments $[11-15]$. In recent years, there have also been extensive efforts [16-19] in studying polarized inelastic scattering of electrons from polarized ${ }^{3} \mathrm{He}$ targets in the deep inelastic and resonance regions aiming at understanding the underlying neutron spin structure. The extraction of the neutron spin structure functions from these experiments requires detailed knowledge of the ${ }^{3} \mathrm{He}$ nuclear ground-state structure $[6,20,21]$.

However, to probe the nuclear ground-state structure, to extract the neutron electromagnetic form factors, or to extract the neutron spin structure function in the resonance region, the reaction mechanism, especially FSI and meson-exchange currents (MEC) effects, must be well understood. Recently, a nonrelativistic Faddeev calculation which includes both FSI and MEC has been carried out [4] for the first time and describes very well the recent precision data [12] on the transverse asymmetry $A_{T^{\prime}}$ near the top of the quasielastic peak from the ${ }^{3} \mathrm{He}\left(\vec{e}, e^{\prime}\right)$ process at low $Q^{2}$. However, since FSI and MEC effects are relatively small in this region, it is highly desirable to study another region where these two effects are larger to provide a more stringent constraint on the theory. The threshold region of ${ }^{3} \overrightarrow{\mathrm{He}}\left(\vec{e}, e^{\prime}\right)$, which extends from the two-body breakup threshold (with breakup energy of $5.5 \mathrm{MeV}$ ), the three-body breakup threshold (with breakup energy of $7.7 \mathrm{MeV}$ ) to the low energy transfer side of the quasielastic peak, is an ideal place for such a study. First, FSI effects are expected to be large in the threshold region since the final-state nucleons have less kinetic energy and thus have a higher probability of interacting with each other. Second, it has been shown that a substantial contribution from MEC is needed to describe the measured elastic electromagnetic form factors of the three-body system [22]. Therefore one would expect a large MEC effect in the threshold region as well.

A precision measurement of spin observables in the threshold region of ${ }^{3} \overrightarrow{\mathrm{He}}\left(\vec{e}, e^{\prime}\right)$ would thus provide us with important information on the reaction mechanism, thereby placing significant constraints on the theoretical uncertainties in probing the ${ }^{3} \mathrm{He}$ ground-state structure and in extracting the neutron electromagnetic form factors from electron scattering from ${ }^{3} \mathrm{He}$. In this Letter we report the first precision measurement of the spin-dependent asymmetry in the threshold region of ${ }^{3} \mathrm{He}\left(\vec{e}, e^{\prime}\right)$.
For inclusive scattering of longitudinally polarized electrons from a polarized spin- $1 / 2$ target such as ${ }^{3} \mathrm{He}$, the spin-dependent asymmetry is defined as $A=\frac{\sigma^{h+}-\sigma^{h-}}{\sigma^{h+}+\sigma^{h-}}$, where $\sigma^{h^{ \pm}}$are the cross sections for the two different helicities of the polarized electrons. It is given in terms of the quasielastic response functions as [23]

$$
A=\frac{-\left(\cos \theta^{*} \nu_{T^{\prime}} R_{T^{\prime}}+2 \sin \theta^{*} \cos \phi^{*} \nu_{T L^{\prime}} R_{T L^{\prime}}\right)}{\nu_{L} R_{L}+\nu_{T} R_{T}},
$$

where the $\nu_{k}$ are kinematic factors and $\theta^{*}$ and $\phi^{*}$ are the polar and azimuthal angles of target spin with respect to the three-momentum transfer vector $\mathbf{q}$ in the laboratory frame. $R_{L}$ and $R_{T}$ are the spin-independent longitudinal and transverse response functions, while $R_{T^{\prime}}$ and $R_{T L^{\prime}}$ are the spin-dependent transverse and longitudinal-transverse ones. The response functions depend on the electron energy transfer $\omega$ and the four-momentum transfer squared $Q^{2}$. By choosing $\theta^{*}=0^{\circ}\left(90^{\circ}\right)$, one selects the transverse asymmetry $A_{T^{\prime}}$ (longitudinal-transverse asymmetry $A_{T L^{\prime}}$ ).

The experiment was carried out in Hall A at the Thomas Jefferson National Accelerator Facility (JLab), using a longitudinally polarized continuous wave electron beam of $10 \mu \mathrm{A}$ current incident on a high-pressure polarized ${ }^{3} \mathrm{He}$ gas target. A detailed description of this experiment can be found in a previous publication [12].

Electrons scattered from the target were detected in the two Hall A high resolution spectrometers, HRSe and HRSh. The data from HRSe have been presented in a previous Letter [12]. The data from HRSh were used for this analysis and covered both the elastic peak and the threshold region. Since the elastic asymmetry can be calculated accurately at low $Q^{2}$ using the well-known elastic form factors of ${ }^{3} \mathrm{He}$ [24], the elastic measurement allows a precise monitoring of the product of the beam and target polarizations, $P_{b} P_{t}$. Two kinematic points were measured in the threshold region, one with a central $Q^{2}$ value of $0.1(\mathrm{GeV} / c)^{2}$ at an incident beam energy $E_{0}=0.778 \mathrm{GeV}$ and the other with a central $Q^{2}$ value of $0.2(\mathrm{GeV} / c)^{2}$ at $E_{0}=1.727 \mathrm{GeV}$. The target spin was oriented at $62.5^{\circ}$ to the right of the incident electron momentum direction, while the outgoing electron momentum directions were $23.7^{\circ}$ and $15.0^{\circ}$ to the right of the incident electron momentum direction for $Q^{2}=0.1$ and $0.2(\mathrm{GeV} / c)^{2}$, respectively. This corresponds to $\theta^{*}$ from $131.2^{\circ}$ to $136.5^{\circ}$ for $Q^{2}=0.1(\mathrm{GeV} / c)^{2}$, and from $134.2^{\circ}$ to $140.0^{\circ}$ for $Q^{2}=0.2(\mathrm{GeV} / c)^{2}$.

The yield for each electron helicity state was corrected by its corresponding charge and computer dead time, and the raw experimental asymmetry was formed as a function of the excitation energy in the ${ }^{3} \mathrm{He}$ system, which is defined as $E_{x}=\sqrt{M^{2}+2 M \omega-Q^{2}}-M$, where $M$ is the mass of the ${ }^{3} \mathrm{He}$ target. A $5 \mathrm{MeV}$ bin was used for the excitation energy. The range of the excitation energy is from $5.5 \mathrm{MeV}$, which corresponds to the two-body breakup threshold, to about $35 \mathrm{MeV}$ for $Q^{2}=0.1(\mathrm{GeV} / c)^{2}$, and about $50 \mathrm{MeV}$ for $Q^{2}=0.2(\mathrm{GeV} / c)^{2}$. The raw asymmetry was then corrected for dilutions due to scattering from 
the target walls, the nitrogen admixture inside the target cell, and $P_{b} P_{t}$. The physics asymmetry was obtained after subtraction of the elastic radiative tail contribution, radiative correction of the quasielastic asymmetry, and correction for spectrometer acceptance and bin-averaging effects, all obtained from a Monte Carlo simulation [25]. The external radiative correction was treated following the standard procedure of Mo and Tsai [26]. The internal radiative correction was calculated using the covariant formalism of Akushevich and Shumeiko [27]. This procedure requires knowledge of ${ }^{3} \mathrm{He}$ nuclear response functions at various kinematics points, which were obtained from full Faddeev calculations [4].

Results for the physics asymmetry at both kinematics are shown in Fig. 1. The uncertainty in determining the excitation energy is about $0.4 \mathrm{MeV}$ at $Q^{2}=0.1(\mathrm{GeV} / c)^{2}$, and $1.0 \mathrm{MeV}$ at $Q^{2}=0.2(\mathrm{GeV} / c)^{2}$, dominated by the uncertainty in the beam energy. The vertical error bars on the data are the statistical and systematic errors added in quadrature. The systematic uncertainty includes contributions from the determination of $P_{b} P_{t}$, target wall and $N_{2}$
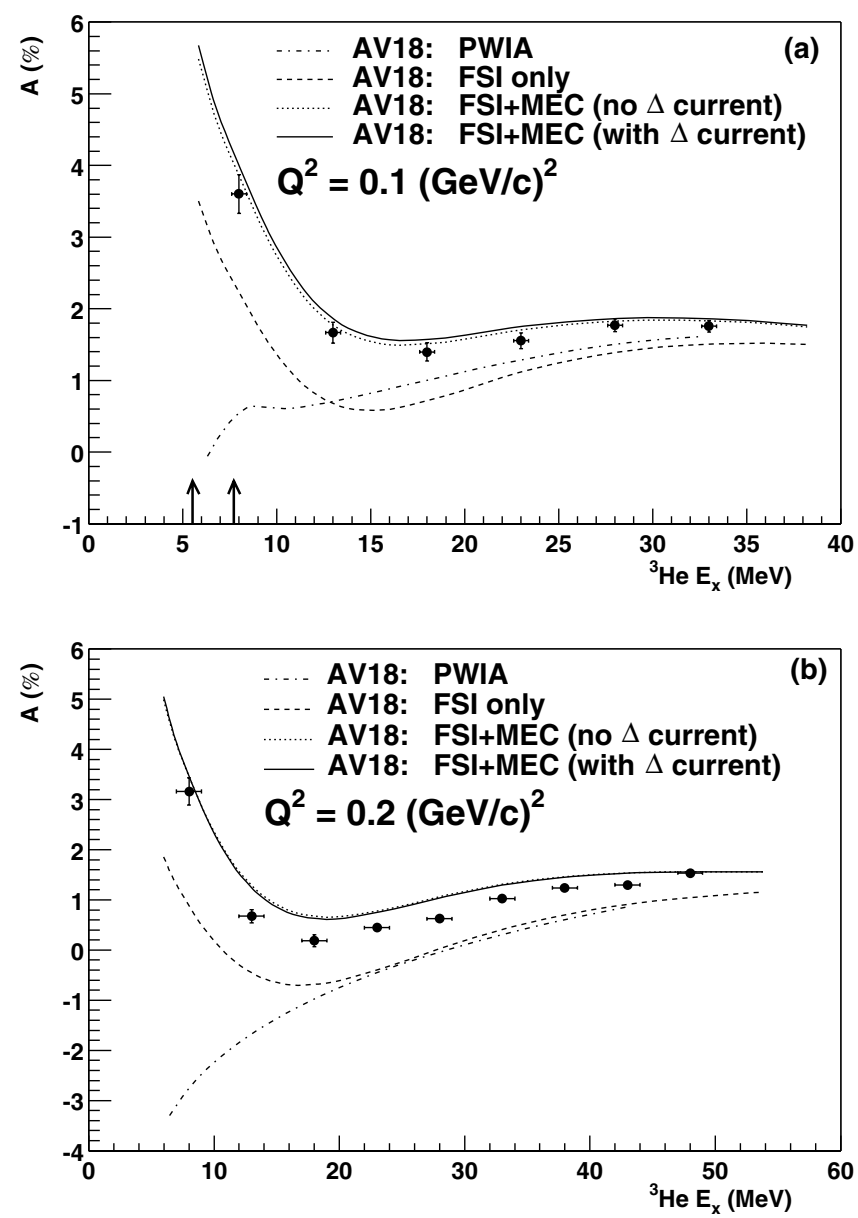

FIG. 1. The physics asymmetry together with theoretical calculations for (a) $Q^{2}=0.1(\mathrm{GeV} / c)^{2}$ and (b) $Q^{2}=$ $0.2(\mathrm{GeV} / c)^{2}$. The theoretical calculations are all performed using AV 18 potential, but with different reaction mechanisms. The arrows point to the two-body and three-body breakup thresholds. background subtraction, elastic radiative tail subtraction, radiative correction, and the correction of spectrometer acceptance and bin-averaging effects. A careful analysis of systematic uncertainties was carried out and the results are shown together with the physics asymmetry and statistical uncertainties in Table I for $Q^{2}=0.1(\mathrm{GeV} / c)^{2}$ and Table II for $Q^{2}=0.2(\mathrm{GeV} / c)^{2}$.

All theoretical calculations were performed using AV18 [28] as the NN interaction potential and the Höhler nucleon form factor parametrization [29]. Plane wave impulse approximation (PWIA) calculations [9,30] are shown as dot-dashed lines. Nonrelativistic Faddeev calculations with FSI only [31] are shown as dashed lines. Nonrelativistic Faddeev calculations which include both FSI and MEC [31] are shown as dotted lines without the inclusion of the $\Delta$ isobar current, and solid lines with the inclusion of the $\Delta$ isobar current. The MEC's ( $\pi$ and $\rho$ exchanges) were chosen according to a prescription given by Riska [32], which guarantees to a large extent the consistency of the MEC's to the NN force used. The agreement between the full calculation and the data is very good at $Q^{2}=0.1(\mathrm{GeV} / c)^{2}$, and a relatively small discrepancy is observed at $Q^{2}=0.2(\mathrm{GeV} / c)^{2}$.

To investigate the effects of different NN potentials, we compare our data with full Faddeev calculations using the AV18 potential and the Bonn-B potential [33], a nonlocal potential which is very different from the local AV18 potential. The result is shown in Fig. 2. As can be seen, the difference between the theoretical calculations using these two potentials is very small, which suggests that this observable is not sensitive to the choice of different NN potentials and the corresponding exchange currents.

Theoretical uncertainties due to $G_{E}^{p}, G_{M}^{p}, G_{E}^{n}$, and $G_{M}^{n}$ were studied using PWIA [6,34]. The relative difference between the asymmetries calculated with the nucleon form factors from the Höhler parametrization and from data $[12-15,35-38]$ was found to be around $1 \%$, and thus theoretical uncertainties due to nucleon form factors are completely negligible.

The good agreement between the full calculation and the data at $Q^{2}=0.1(\mathrm{GeV} / c)^{2}$ suggests the validity of the

TABLE I. Systematic uncertainties at each excitation energy $\left(E_{x}\right)$ for $Q^{2}=0.1(\mathrm{GeV} / c)^{2}$, which include contributions from the determination of $P_{b} P_{t}\left(\delta^{\mathrm{pol}}\right)$, target wall and $N_{2}$ dilution $\left(\delta^{\text {dil }}\right)$, elastic radiative tail subtraction $\left(\delta^{\text {ert }}\right)$, radiative correction $\left(\delta^{\mathrm{rc}}\right)$, and the correction of spectrometer acceptance and bin-averaging effects $\left(\delta^{\text {acc }}\right)$. The physics asymmetry $(A)$ and statistical uncertainties $\left(\delta^{\text {stat }}\right)$ are also shown.

\begin{tabular}{rcccccc}
\hline \hline $\begin{array}{c}E_{x} \\
(\mathrm{MeV})\end{array}$ & $\begin{array}{c}A \pm \delta^{\text {stat }} \\
(\%)\end{array}$ & $\begin{array}{c}\delta^{\text {pol }} \\
(\%)\end{array}$ & $\begin{array}{c}\delta^{\text {dil }} \\
(\%)\end{array}$ & $\begin{array}{c}\delta^{\text {ert }} \\
(\%)\end{array}$ & $\begin{array}{c}\delta^{\text {rc }} \\
(\%)\end{array}$ & $\begin{array}{c}\delta^{\text {acc }} \\
(\%)\end{array}$ \\
\hline 8.0 & $3.602 \pm 0.157$ & 0.153 & 0.048 & 0.105 & 0.020 & 0.032 \\
13.0 & $1.666 \pm 0.100$ & 0.073 & 0.021 & 0.061 & 0.014 & 0.015 \\
18.0 & $1.399 \pm 0.082$ & 0.050 & 0.012 & 0.076 & 0.010 & 0.009 \\
23.0 & $1.553 \pm 0.071$ & 0.043 & 0.009 & 0.066 & 0.008 & 0.023 \\
28.0 & $1.768 \pm 0.063$ & 0.043 & 0.008 & 0.042 & 0.008 & 0.009 \\
33.0 & $1.756 \pm 0.066$ & 0.039 & 0.007 & 0.016 & 0.010 & 0.009 \\
\hline \hline
\end{tabular}


TABLE II. Systematic uncertainties for $Q^{2}=0.2(\mathrm{GeV} / c)^{2}$. Symbols are the same as in Table I.

\begin{tabular}{rcccccc}
\hline \hline $\begin{array}{c}E_{x} \\
(\mathrm{MeV})\end{array}$ & $\begin{array}{c}A \pm \delta^{\text {stat }} \\
(\%)\end{array}$ & $\begin{array}{c}\delta^{\text {pol }} \\
(\%)\end{array}$ & $\begin{array}{c}\delta^{\text {dil }} \\
(\%)\end{array}$ & $\begin{array}{c}\delta^{\text {ert }} \\
(\%)\end{array}$ & $\begin{array}{c}\delta^{\text {rc }} \\
(\%)\end{array}$ & $\begin{array}{c}\delta^{\text {acc }} \\
(\%)\end{array}$ \\
\hline 8.0 & $3.161 \pm 0.170$ & 0.121 & 0.070 & 0.121 & 0.018 & 0.014 \\
13.0 & $0.676 \pm 0.094$ & 0.044 & 0.022 & 0.064 & 0.034 & 0.011 \\
18.0 & $0.190 \pm 0.071$ & 0.022 & 0.010 & 0.036 & 0.075 & 0.035 \\
23.0 & $0.446 \pm 0.058$ & 0.020 & 0.008 & 0.021 & 0.012 & 0.021 \\
28.0 & $0.625 \pm 0.049$ & 0.019 & 0.006 & 0.012 & 0.006 & 0.021 \\
33.0 & $1.025 \pm 0.045$ & 0.024 & 0.007 & 0.007 & 0.012 & 0.017 \\
38.0 & $1.241 \pm 0.041$ & 0.026 & 0.007 & 0.005 & 0.015 & 0.013 \\
43.0 & $1.300 \pm 0.041$ & 0.026 & 0.006 & 0.005 & 0.018 & 0.011 \\
48.0 & $1.537 \pm 0.050$ & 0.028 & 0.005 & 0.005 & 0.020 & 0.022 \\
\hline \hline
\end{tabular}

current way of treating FSI and MEC in the full calculation. The small discrepancy at $Q^{2}=0.2(\mathrm{GeV} / c)^{2}$ may be due to the fact that some $Q^{2}$-dependent effects, such as the relativisitic effect, are not included in the current nonrelativistic Faddeev calculation.

In conclusion we have presented the first precision data on the spin-dependent asymmetry in the threshold region of ${ }^{3} \overrightarrow{\mathrm{He}}\left(\vec{e}, e^{\prime}\right)$. The agreement between the data and nonrelativistic Faddeev calculations which include both FSI
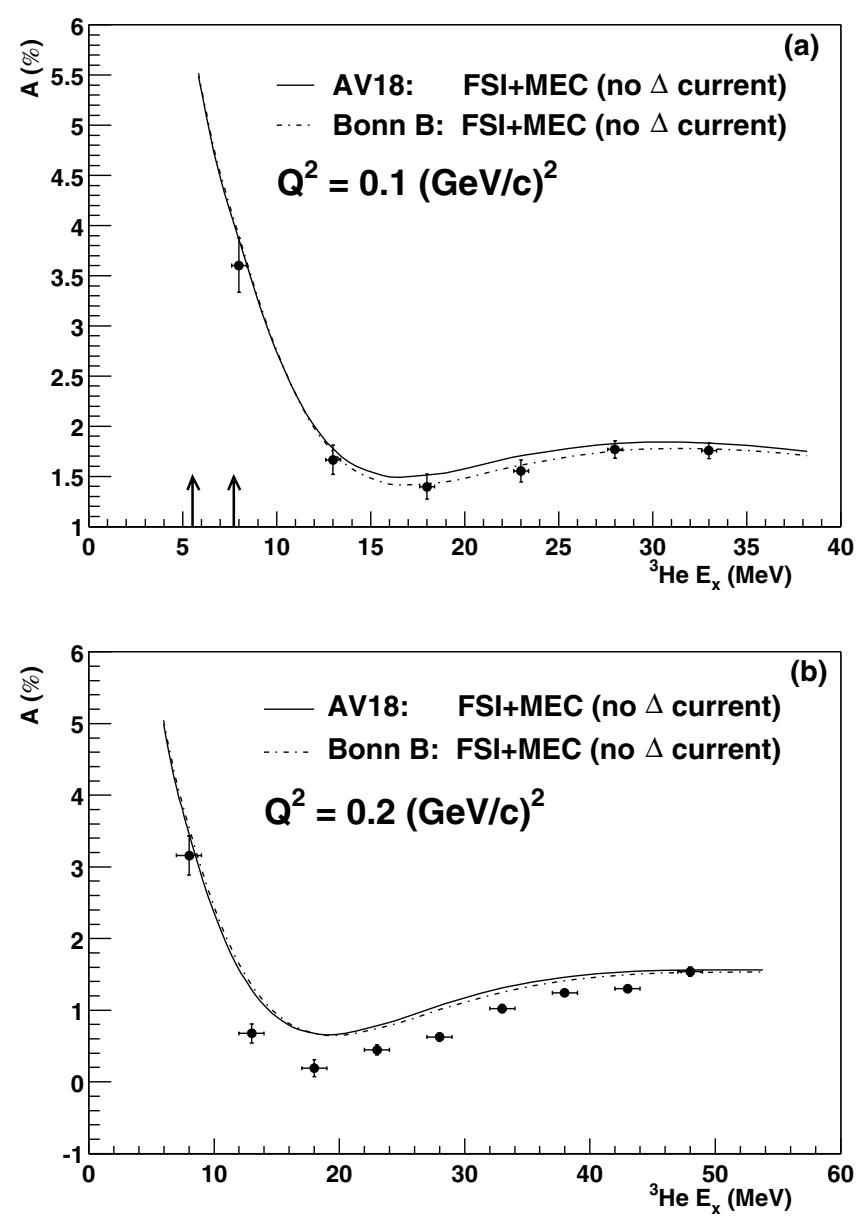

FIG. 2. The physics asymmetry together with two full Faddeev calculations, one using AV18 as the NN potential, the other using Bonn-B: (a) $Q^{2}=0.1(\mathrm{GeV} / c)^{2}$ and (b) $Q^{2}=0.2(\mathrm{GeV} / c)^{2}$. and MEC effects is very good at $Q^{2}=0.1(\mathrm{GeV} / c)^{2}$, while the discrepancy at $Q^{2}=0.2(\mathrm{GeV} / c)^{2}$ might be due to some $Q^{2}$-dependent mechanism.

We thank the Hall A technical staff and the Jefferson Lab Accelerator Division for their outstanding support during this experiment. We also thank T.W. Donnelly for many helpful discussions. This work was supported in part by the U.S. Department of Energy, DOE/EPSCoR, the U.S. National Science Foundation, the Science and Technology Cooperation Germany-Poland and the Polish Committee for Scientific Research, the Ministero dell'Università e della Ricerca Scientifica e Tecnologica (Murst), the French Commissariat à l'Énergie Atomique, Centre National de la Recherche Scientifique (CNRS), Conseil Régional d'Auvergne, the Italian Istituto Nazionale di Fisica Nucleare (INFN), and a grant of European Foundation Project No. INTAS-99-0125. This work was supported by DOE Contract No. DE-AC05-84ER40150 under which the Southeastern Universities Research Association (SURA) operates the Thomas Jefferson National Accelerator Facility. The numerical calculations were performed on the PVP machines at the U.S. National Energy Research Scientific Computer Center (NERSC) and the CRAY T90 of the NIC in Jülich.

[1] W. Glöckle et al., Phys. Rep. 274, 107 (1996).

[2] H. Anklin et al., Nucl. Phys. A636, 189 (1998).

[3] J. Golak et al., Phys. Rev. C 52, 1216 (1995).

[4] J. Golak et al., Phys. Rev. C 62, 054005 (2000).

[5] B. Blankleider et al., Phys. Rev. C 29, 538 (1984).

[6] J. L. Friar et al., Phys. Rev. C 42, 2310 (1990).

[7] C. Ciofi degli Atti et al., Phys. Rev. C 46, R1591 (1992).

[8] R.-W. Schulze and P. U. Sauer, Phys. Rev. C 48, 38 (1993).

[9] C. Ciofi degli Atti et al., Phys. Rev. C 51, 1108 (1995).

[10] S. Ishikawa et al., Phys. Rev. C 57, 39 (1998).

[11] V. V. Kotlyar et al., Few-Body Syst. 28, 35 (2000).

[12] W. Xu et al., Phys. Rev. Lett. 85, 2900 (2000).

[13] M. Meyerhoff et al., Phys. Lett. B 327, 201 (1994).

[14] J. Becker et al., Eur. Phys. J. A 6, 329 (1999).

[15] D. Rohe et al., Phys. Rev. Lett. 83, 4257 (1999).

[16] P. L. Anthony et al., Phys. Rev. Lett. 71, 959 (1993); P. L. Anthony et al., Phys. Rev. D 54, 6620 (1996).

[17] K. Abe et al., Phys. Rev. Lett. 79, 26 (1997); K. Abe et al., Phys. Lett. B 404, 377 (1997).

[18] K. Ackerstaff et al., Phys. Lett. B 404, 383 (1997).

[19] Z.-E. Meziani et al., Jefferson Lab Proposal No. E94-010; J. P. Chen et al., Jefferson Lab Proposal No. E97-110.

[20] C. Ciofi degli Atti et al., Phys. Rev. C 48, R968 (1993).

[21] C. Ciofi degli Atti et al., Phys. Lett. B 404, 223 (1997).

[22] A. Amroun et al., Phys. Rev. Lett. 69, 253 (1992).

[23] T. W. Donnelly and A.S. Raskin, Ann. Phys. (N.Y.) 169, 247 (1986).

[24] A. Amroun et al., Nucl. Phys. A579, 596 (1994); C. R. Otterman et al., Nucl. Phys. A435, 688 (1985); P. C. Dunn et al., Phys. Rev. C 27, 71 (1983).

[25] F. Xiong et al. (to be published).

[26] L. W. Mo and Y.-S. Tsai, Rev. Mod. Phys. 41, 205 (1969). 
[27] I. V. Akushevich and N. M. Shumeiko, J. Phys. G 20, 513 (1994).

[28] R. B. Wiringa et al., Phys. Rev. C 51, 38 (1995).

[29] G. Höhler et al., Nucl. Phys. B114, 505 (1976).

[30] A. Kievsky et al., Phys. Rev. C 56, 64 (1997).

[31] J. Golak et al., Phys. Rev. C 63, 034006 (2001).

[32] D. O. Riska, Phys. Scr. 31, 107 (1985).
[33] R. Machleidt, Adv. Nucl. Phys. 19, 189 (1989).

[34] J.-O. Hansen et al., Phys. Rev. Lett. 74, 654 (1995).

[35] T. Eden et al., Phys. Rev. C 50, R1749 (1994).

[36] M. Ostrick et al., Phys. Rev. Lett. 83, 276 (1999).

[37] C. Herberg et al., Eur. Phys. J. A 5, 131 (1999).

[38] I. Passchier et al., Phys. Rev. Lett. 82, 4988 (1999). 